Themenheft Nr. 40: CoViD-19 und die digitale Hochschulbildung. Irritationen, Einsichten und Programmatiken

Herausgegeben von Markus Deimann, Marios Karapanos und Klaus Rummler

\title{
Zuhause statt Hörsaal
}

\section{Erfahrungen und Einschätzungen von Hochschulangehörigen zur Umstellung der Lehre im ersten pandemie-bedingten Lockdown der Universitäten}

\author{
Katharina Neuber ${ }^{1}$ (D) und Kerstin Göbel ${ }^{1}$ (D) \\ ${ }^{1}$ Universität Duisburg-Essen
}

\section{Zusammenfassung}

Die Digitalisierung von Studium und Lehre stellt einen komplexen Entwicklungsprozess dar, der für alle beteiligten Akteurinnen und Akteure Herausforderungen, wie beispielsweise die Auseinandersetzung mit den Einsatzmöglichkeiten digitaler Medien und die Entwicklung digitaler Kompetenzen, mit sich bringt (Getto und Schulenburg 2018; Kerres 2016). Der Beginn der Corona-Pandemie und die damit verbundene erstmalige Schliessung der Universitäten im Frühjahr 2020 erforderte innerhalb kürzester Zeit die Umstellung der konventionellen universitären Lehre in Präsenzform auf online-gestützte Lehrformate. Wie diese Umstellung von Hochschulangehörigen in Deutschland erlebt wurde, in welchem Ausmass digitale Tools in der eigenen Lehre im Sommersemester 2020 eingesetzt wurden und welche personenbezogenen Merkmale hierfür relevant erscheinen, ist Gegenstand des vorliegenden Beitrags. Die deskriptive Auswertung der bundesweiten Befragung von Dozierenden deutscher Hochschulen verdeutlicht ambivalente Erfahrungen mit der Umstellung auf Online-Lehre. Dennoch wird insgesamt die Bewältigung der pädagogisch-didaktischen Herausforderungen durch die zeitweilige Schliessung der Universitäten aus Sicht der Dozierenden als erfolgreich eingeschätzt. Für das Ausmass der Nutzung digitaler Tools während des Lockdowns zeigen sich in den Korrelationsanalysen signifikante Zusammenhänge mit den bisherigen Vorerfahrungen, der selbsteingeschätzten Fähigkeit, digital zu lehren, sowie Unterschiede zwischen männlichen und weiblichen Befragten. 
At Home Instead of Lecture Hall. Experiences of University Teachers on the Transition to Online Teaching in the First Pandemic-Related Lockdown of Universities

\begin{abstract}
The digitization of studies and teaching represents a complex development process with challenges for all actors involved, such as dealing with the possible uses of digital media and the development of digital competencies (Getto and Schulenburg 2018; Kerres, 2016). The onset of the Corona pandemic and the associated first-time closure of universities in spring 2020 required the transition from conventional university teaching in faceto-face form to online-supported teaching formats within a very short time. How this transition was experienced by university staff in Germany, to what extent digital tools were used in their own teaching during the summer semester 2020 and which personal characteristics appear to be relevant for this is the subject of this paper. The descriptive analysis of the nationwide survey of teachers at German universities reveals ambivalent experiences with the transition to online teaching. Nevertheless, from the point of view of the university teachers, the overcoming of the pedagogical-didactic challenges caused by the temporary closure of the universities is assessed as successful overall. For the extent of the use of digital tools during the first lockdown, significant correlations with previous experience, self-assessed ability to teach digitally, and differences between male and female respondents emerge in the correlation analyses.
\end{abstract}

\title{
1. Einleitung
}

Die Verbreitung des Coronavirus im Frühjahr 2020 wirkte sich auf unsere Gesellschaft in vielfältiger Weise aus. Zur Eindämmung des Virus wurden weltweit Massnahmen erlassen, von denen der Bildungssektor nicht ausgenommen war (Education International 2020; Flores und Gago 2020). Die Schliessung öffentlicher Einrichtungen und die erweiterten Kontaktbeschränkungen - unter dem Begriff «Lockdown»zusammengefasst - führten dazu, dass weltweit mehr als 87 \% der Schülerinnen und Schüler fortan Zuhause statt in der Schule lernen mussten (UNESCO 2020). Wird die Digitalisierung von Bildungsprozessen in der Literatur als komplexer Entwicklungsprozess beschrieben, der alle Beteiligten vor Herausforderungen, wie z. B. die Auseinandersetzung mit den Einsatzmöglichkeiten digitaler Medien, stellt (Getto und Schulenburg 2018; Kerres 2016), erforderte die erste Schliessung der Bildungseinrichtungen im Zuge der Pandemie die Reorganisation schulischer und hochschulischer Lehr-Lern-Prozesse binnen kürzester Zeit. 
Im vorliegenden Beitrag werden Befunde einer ländervergleichenden Studie ${ }^{1}$ präsentiert, die sich den Erfahrungen von Hochschullehrenden mit der Umstellung der konventionellen Präsenzlehre auf online-gestützte Lehr-Lernformate (OnlineLehre) im Sommersemester 2020 widmet. Während erste Projektergebnisse bereits eine positive Bewältigung der pandemie-bedingten Herausforderungen am Hochschulstandort Duisburg-Essen verdeutlichten (Göbel et al. 2021), ermöglichen die vorliegenden Befunde einer Stichprobe von deutschen Hochschullehrenden unterschiedlicher Universitäten eine institutionsunabhängige Betrachtung der Erfahrungen mit der Umstellung der Lehre.

\subsection{Digitalisierung von Studium und Lehre}

Im Bildungssektor wurde die Digitalisierung von Studium und Lehre bereits vor Ausbruch der Corona-Pandemie von politischer Seite eingefordert (Bundesregierung 2018) und hat auf Ebene der Hochschulleitungen an Interesse gewonnen (z. B. Gilch et al. 2019; Kerres 2016; van Ackeren et al. 2017). In Ergänzung zur Präsenzlehre birgt der Einsatz digitaler Medien das Potenzial, neue Methoden und Lernwerkzeuge zu erschliessen und bisherige Lerngelegenheiten und -materialien anzureichern. Dadurch können Lerninhalte auf unterschiedliche Weise präsentiert und neue Kommunikations- und Kollaborationsformen ausprobiert werden (Müller, Eichhorn, und Tillmann 2019). Der Einsatz digitaler Tools wird auch als Chance gesehen, der Vielfalt der Bedarfe von Studierenden zu begegnen, indem asynchrone Formate wie Videos oder aufgezeichnete Präsentationen zeit- und ortsunabhängig genutzt werden können (Kümmel et al. 2020). Der Einsatz digitaler Tools in der Hochschule ermöglicht individualisierte und flexible Lernerfahrungen und kann dadurch die didaktische Qualität der Lehre und den Kompetenzerwerb der Studierenden befördern (Getto, Hintze, und Kerres 2018; Gilch et al. 2019; Wannemacher et al. 2016).

Mittlerweile stehen eine Vielzahl an digitalen Werkzeugen und Tools für den Einsatz in der Hochschullehre zur Verfügung, die entweder über einen bestimmten Zeitraum (asynchron) oder in «Echtzeit» (synchron) genutzt werden können (Wannemacher et al. 2016). Beispielsweise können durch die Einbindung von Learning Management System (LMS)-Plattformen asynchrone Werkzeuge wie Chat- und Diskussionsforen, Podcasts oder Videos bereitgestellt und zeitlich flexibel bearbeitet werden. Demgegenüber stellen Webkonferenzsysteme (z. B. Webinar, Videokonferenz) synchrone Werkzeuge für Online-Lehre dar, die Hochschullehrende und -lernende zeitgleich,

1 An der ländervergleichenden Studie CRTS (Coronavirus-Related Teaching Situation) beteiligen sich Forscherinnen und Forscher folgender Institutionen: Hebräische Universität Jerusalem (Israel), Universität Basel (Switzerland), Universidad de Buenos Aires (Argentina), Universität Duisburg-Essen (Germany) und Universität Miami (USA). Inzwischen gibt es weitere Beteiligungen durch Forscherinnen und Forschern der Universidad Tecnológica Nacional (Argentina), Universidad Autónoma (Chile), University of Strasbourg (France), University of Upper Alsace (France) und der Westminster University (UK). Nähere Informationen zum Projekt finden sich unter der URL: https://www.uni-due.de/unterrichtsentwicklung/crts. 
aber ortsunabhängig nutzen können. Vor Ausbruch der Corona-Pandemie war reine Online-Lehre im Hochschulsektor kaum vorzufinden (Handke 2020; Wannemacher et al. 2016). Die Anreicherung der Präsenzlehre durch den Einsatz digitaler Elemente, z. B. die Verwendung von Präsentationstools wie PowerPoint, und die Nutzung von LMS-Plattformen zur Bereitstellung von Bibliografien, elektronischer Dokumente und Materialien (Schmid et al. 2017; Riedel und Börner 2016), gehörten hingegen zum Standard vieler Hochschulen. Auch Videos (z. B. Erklär- und Lehrvideos oder ELectures) waren vielfältig in der Hochschullehre integriert (Schmid et al. 2017), wobei von Lehrenden zumeist fremdproduzierte Tutorials und Erklärvideos mit dem Ziel der Wiederholung und Vertiefung der in Präsenzphasen vermittelten Fachinhalte eingesetzt wurden, während synchrone Formate wie Webinare, Videokonferenzen oder Livestreaming eine eher untergeordnete Rolle spielten (Schaarschmidt, Albrecht, und Börner 2016). Obwohl sich in den Hochschulkonzepten vor dem Hintergrund von beispielsweise Inverted-Classroom-Ansätzen, bei denen Lehrinhalte zunächst virtuell vermittelt (z. B. durch die Rezeption von Videos, eingebettet in LMS-Plattformen) und in anknüpfenden Präsenzphasen vertieft werden, in den vergangenen Jahren ein steigendes Interesse an didaktischen Selbstlernszenarien abzeichnete (Müller, Eichhorn, und Tillmann 2019), wurden derartige Formate im Vergleich zu Präsentationstools oder LMS-Plattformen von Hochschullehrenden deutlich seltener eingesetzt. Dies mag mitunter daran liegen, dass Lehrende dem alleinigen Einsatz von Videos zur Wissensvermittlung eher skeptisch gegenüberstehen (Schmid et al. 2017). Dieser Befund unterstützt das Technologieakzeptanzmodell nach Davis $(1989,1993)$, wonach die persönliche Einstellung gegenüber der Nutzung einer Technologie entscheidend für die tatsächliche Nutzungsabsicht ist. Für die Einstellung und folglich Nutzungsabsicht scheint zum einen die mit einer Technologie verbundene Nützlichkeit (Verbesserung der eigenen Arbeitsleistung), zum anderen die wahrgenommene Benutzerfreundlichkeit (im Sinne einer mühelosen Verwendung) relevant. Je grösser die Nützlichkeit und je einfacher die Bedienbarkeit einer Technologie wahrgenommen werden, desto eher sind Anwendende bereit, das jeweilige System zu nutzen (vgl. auch Teo 2009). In diesem Zusammenhang spielen vermutlich auch Aspekte der Selbstwirksamkeitserwartung eine Rolle (Bandura 1977); Personen mit einer erhöhten Selbstwirksamkeitserwartung in Bezug auf den Umgang mit einer Technologie werden diese wahrscheinlich als leichter zu benutzen wahrnehmen (Davis 1989) und eine höhere Nutzungsbereitschaft zeigen (Teo 2009). Empirische Befunde weisen in diese Richtung und zeigen, dass für den Einsatz digitaler Tools in der Hochschullehre Wirksamkeitserwartungen und Fähigkeiten der Dozierenden im Umgang mit Technologien (Buchanan, Sainter, und Saunders 2013; Jokiaho und May 2017) oder die empfundene Nützlichkeit dieser (Marzilli et al. 2014) von Bedeutung sind. 
Die Digitalisierung von Studium und Lehre stellt einen komplexen Entwicklungsprozess dar, der neben einer offenen Haltung und digitalen Kompetenzen der Beteiligten auch das Vorhandensein zeitlicher Ressourcen zur Vorbereitung des Einsatzes digitaler Medien sowie die technische Infrastruktur und institutionelle Unterstützung erfordert (Getto, Hintze, und Kerres 2018; Kerres 2016; Müller, Eichhorn, und Tillmann 2019). Insbesondere fehlende digitale Kompetenzen und eine geringe Benutzerfreundlichkeit digitaler Tools stellen nicht zu unterschätzende Nutzungshindernisse dar (Jokiaho und May 2017; Marzilli et al. 2014). Daher können Qualifizierungsund Beratungsangebote hilfreich sein. Befunde zur Digitalisierung an Hochschulen weisen darauf hin, dass insbesondere weibliche Dozierende derartige Angebote für wichtig erachten (Riedel und Börner 2016). Untersuchungen zur Digitalisierung in Schulen signalisieren ebenfalls Unterschiede zwischen den Geschlechtern (Drossel et al. 2019): Während sich männliche Lehrkräfte häufiger als kompetent einschätzen, mit Technologien umzugehen und zudem regelmässig auf digitale Medien zurückzugreifen, setzen weibliche und ältere Lehrpersonen digitale Medien signifikant seltener im Unterricht ein.

\subsection{Hochschullehre in Zeiten der Corona-Pandemie}

Auf die erste Schliessung der Hochschulen im Frühjahr 2020 mussten die Hochschulangehörigen binnen kürzester Zeit reagieren. Unter hohem Zeitdruck wurden digitale Lehrveranstaltungen implementiert, um das Bildungsangebot aufrecht zu erhalten. Diese kriseninduzierte Übertragung konventioneller Lehre in online-gestützte Formate - mittlerweile unter dem Begriff des «Emergency Remote Teaching (ERT)» (Hodges et al. 2020) bekannt - ist von didaktisch sorgfältig geplanten digitalen Lehrformaten deutlich abzugrenzen, da sie primär das Ziel verfolgte, den hemmenden Effekten der Pandemie auf die Qualität hochschulischer Bildungsprozesse schnellstmöglich entgegenzuwirken und alternative Zugänge zu Lehr-Lerninhalten und mentorieller Begleitung zu ermöglichen (Bozkurt und Sharma 2020).

Zur Unterstützung der Umstellung wurden weltweit Erfahrungsberichte und Leitfäden zur Gestaltung digitaler Lehre sowie zur Nutzung unterschiedlicher Tools erstellt (z. B. Bao 2020; Czerniewicz et al. 2020; Ferdig et al. 2020; Lowenthal et al. 2020; Moorhouse 2020; Toquero 2020; Zhu und Liu 2020). Vielerorts wurden im Rahmen des ersten Lockdowns zudem Befragungen mit Studierenden und Dozierenden realisiert, die deren Einschätzungen hinsichtlich technischer Ausstattung, Qualität der digitalen Lehre sowie damit verbundene Unterstützungsbedarfe adressieren (z. B. Dittler und Kreidl 2021; Göbel et al. 2021; Karapanos et al. 2021; Mulders und Krah 2021; Stammen und Ebert 2020; Zierer 2020). Die bisherigen Befunde verdeutlichen in der Zusammenschau, dass das erste digitale Semester überwiegend erfolgreich verlaufen ist (Almazova et al. 2020; Kehrer und Thillosen 2021; Watermeyer et al. 
2020). Auch erste Ergebnisse der ländervergleichenden CRTS-Studie (CoronavirusRelated Teaching Situation-Study) signalisieren, dass die Umstellung auf digitale Lehr-Formate im Frühjahr 2020 aus Sicht der befragten Dozierenden aus Deutschland, der Schweiz, Israel und Argentinien erfolgreich bewältigt wurde (Göbel et al. 2021; Dorfsman und Horenczyk 2021; Lion, Cukierman, und Scardigli eingereicht). Wenngleich die Dozierenden je nach institutioneller Zugehörigkeit unterschiedliche Vorerfahrungen mit dem Einsatz digitaler Tools in der Hochschullehre aufweisen (Göbel et al. 2021; Lion, Cukierman, und Scardigli eingereicht) und Online-Lehre grundsätzlich als weniger zufriedenstellend erleben (vgl. Dorfsman und Horenczyk 2021), schätzen sie sowohl ihre Kompetenzen, online zu lehren, als auch die Umsetzung der digitalen Lehre während des ersten Lockdowns der Universitäten optimistisch ein. Ein wichtiger Unterschied zur Präsenzlehre betrifft insbesondere die Art und Weise, wie Lernende in digitale Lehrveranstaltungen eingebunden werden und in Austausch treten können. So wurde im Rahmen der Befragungen im CRTSProjekt von den Hochschullehrenden der fehlende Austausch mit den Studierenden während des ersten Lockdowns der Hochschulen als einschränkend erlebt. Analog hierzu verdeutlichen die Befunde bisheriger Studierendenbefragungen, dass der gestiegene Arbeitsaufwand und fehlende Kontakt zu Dozierenden und Peers auch von Studierenden bemängelt (Brunner 2021; Karapanos et al. 2021; Stammen und Ebert 2020; Zierer 2020) und die Beteiligung in synchron angebotenen digitalen Lehrveranstaltungen im Vergleich zur Präsenzlehre als schwieriger empfunden wird (Dittler und Kreidl 2021). Nichtsdestotrotz nehmen Studierende auch vielfältige Potenziale der Umstellung auf Online-Lehre wahr, z. B. Flexibilität und Autonomie beim Lernen sowie digitale Kompetenzentwicklung (Brunner 2021; Greimel-Fuhrmann et al. 2021; Karapanos et al. 2021; Mulders und Krah 2021).

\section{2. $\quad$ Fragestellungen}

Vor Ausbruch der Corona-Pandemie gehörte der Einsatz digitaler Präsentationstools und LMS-Plattformen zum Standard vieler Hochschulen in Deutschland, wohingegen ausschliessliche Online-Lehre wenig verbreitet war (Handke 2020). Die sofortige Umstellung auf Online-Lehre bedingt durch den ersten Lockdown der Universitäten im Sommersemester 2020 stellte entsprechend für alle Hochschulangehörigen eine Herausforderung dar. Von besonderem Interesse erscheinen daher die Fragen, wie Dozierende deutscher Hochschulen die Umstellung ihrer Lehre erlebt haben, wie sie den Erfolg dieser Umstellungserfahrung einschätzen und wie sich die Nutzung digitaler Tools in der Lehre verändert hat. Bisherige Forschungsbefunde verdeutlichen die Relevanz zeitlicher Ressourcen, technologischer Infrastruktur, Kompetenzen im Umgang und wahrgenommener Nützlichkeit für den Einsatz digitaler Tools (Davis 1989; Jokiaho und May 2017). Alter und Geschlecht können hierfür ebenso eine Rolle 
spielen (Drossel et al. 2019). Die Umstellung auf digitale Lehre in Zeiten der Pandemie war plötzlich und unausweichlich; es stellt sich daher die Frage, inwieweit Merkmale der Hochschullehrenden auch in dieser Spezialsituation von Bedeutung sein können. Für den vorliegenden Beitrag werden die folgenden Fragestellungen explorativ untersucht:

1. Wie haben die Dozierenden die Umstellung von der konventionellen Lehre in Präsenzform auf Online-Lehre erlebt und wie schätzen sie den Erfolg der ersten online-gestützten Lehreinheiten im Sommersemester 2020 ein?

2. Wie hat sich das Ausmass der Nutzung digitaler Tools für die Lehre während des Coronavirus-bedingten ersten Lockdowns der Universitäten verändert?

3. Zeigen sich Zusammenhänge zwischen den selbsteingeschätzten Kompetenzen zur Umsetzung von Online-Lehre und dem Nutzungsverhalten in Bezug auf digitale Tools? Inwieweit unterscheiden sich Dozierende mit unterschiedlichen personenbezogenen Merkmalen (Geschlecht und Alter) in ihrer Kompetenzeinschätzung und ihrem Nutzungsverhalten?

\section{Methode}

\subsection{Untersuchungskontext und Stichprobe}

Die Befragung von Dozierenden deutscher Hochschulen erfolgte im Kontext der ländervergleichenden CRTS-Studie, welche die Erfahrungen und Bedürfnisse von Hochschullehrenden zu Beginn der Corona-Pandemie und der damit einhergehenden Umstellung auf Online-Lehre in den Blick nimmt. Hierzu wurden Dozierende in Deutschland, der Schweiz, Frankreich, Grossbritannien, Israel, Argentinien, Chile und den USA online befragt, wie sie mit den pädagogisch-didaktischen Herausforderungen umgehen und welche Unterstützungsbedarfe sie sehen. Die Online-Befragung an deutschen Hochschulen wurde im Juni 2020 an der Universität Duisburg-Essen unter der Leitung von Kerstin Göbel realisiert und mittels LimeSurvey durchgeführt. Die Dozierenden sämtlicher Fachbereiche wurden via E-Mail über die Studie informiert und zur Teilnahme an der Befragung eingeladen.

Insgesamt haben $n=292$ Dozierende (davon 176 weiblich; $60.3 \%$ ) von 42 deutschen Hochschulen den Fragebogen vollständig beantwortet. Hinsichtlich Altersstruktur und durchschnittliche Lehrzeit pro Woche wird eine heterogene Zusammensetzung der Stichprobe deutlich (vgl. Tab. 1); gleiches gilt für den Fachbereich, in dem die Befragten hauptsächlich lehren. 71 Befragte ordnen sich den Geisteswissenschaften zu und 44 den Naturwissenschaften, 75 Dozierende lehren hauptsächlich in den Bildungswissenschaften, 74 Befragte in den Sozialwissenschaften und 
32 Dozierende primär im Bereich Wirtschaftswissenschaften. Weiterhin haben Dozierende aus Medizin, Psychologie, Theologie und den Sprachwissenschaften an der Umfrage teilgenommen.

\begin{tabular}{|l|c|c|}
\hline \multicolumn{2}{|c|}{$n$} & $\%$ \\
\hline Geschlecht & 176 & 60.3 \\
\hline weiblich & 103 & 35.3 \\
\hline männlich & 1 & 0.3 \\
\hline divers & 280 & 95.9 \\
\hline Total & \multicolumn{2}{|l|}{} \\
\hline Alter in Jahren & 1 & 0.3 \\
\hline 25 und jünger & 90 & 30.8 \\
\hline $26-35$ & 78 & 26.7 \\
\hline $36-45$ & 61 & 20.9 \\
\hline $46-55$ & 50 & 17.1 \\
\hline $56-65$ & 7 & 2.4 \\
\hline Älter als 65 & 287 & 98.3 \\
\hline Total & & \\
\hline Wöchentliche Lehrtätigkeit in Stunden & 66 & 22.6 \\
\hline $1-2$ & 93 & 31.8 \\
\hline $3-6$ & 83 & 28.4 \\
\hline $7-11$ & 45 & 15.4 \\
\hline 12 und mehr & 287 & 98.3 \\
\hline Total & & \\
\hline
\end{tabular}

Tab. 1.: Angabe gültiger Prozentwerte zur Beschreibung der Stichprobe.

\subsection{Instrumente und Analyseverfahren}

Die Einschätzungen der Dozierenden zur Umstellung auf Online-Lehre im Sommersemester 2020 wurden online mithilfe eines Fragebogens erfasst, welcher gemeinsam von den am CRTS-Projekt teilnehmenden Teams entwickelt und vom Ethikausschuss der Erziehungswissenschaftlichen Fakultät der Hebräischen Universität genehmigt wurde. Zunächst sollten die Dozierenden erste Gedanken in Bezug auf die Umstellung auf Online-Lehre beschreiben und ihre Umstellungserfahrung zusammenfassend bewerten, wobei diese als «sehr positiv und inspirierend», über «überwiegend positiv und ermutigend» bis hin zu «aufwändig», «frustrierend» oder «überfordernd» eingeschätzt werden konnte. Retrospektiv sollten zudem der Vorbereitungsprozess (z. B. die Aneignung von Webkonferenzsystemen) und die Umsetzung der ersten online-gestützten Lehreinheiten bewertet werden. Die Antwortoptionen reichten von 1 = «sehr einfach» bis 5 «sehr schwierig» (Vorbereitungsprozess) bzw. von $1=$ 
«nicht erfolgreich» bis $5=$ «sehr erfolgreich» (Umsetzung erster Lehreinheiten). In einem weiteren Teil des Fragebogens wurde die Nutzung unterschiedlicher digitaler Tools abgefragt. Hierbei sollten die Dozierenden angeben, in welchem Umfang sie vor Ausbruch der Pandemie und während der erstmaligen Schliessung der Universitäten digitale Tools für ihre Lehre genutzt haben bzw. aktuell nutzen ( 1 = «gar nicht» bis $4=$ «in grossem Umfang»). Abschliessend wurde neben den personenbezogenen Merkmalen Geschlecht und Alter erhoben, inwieweit sich die Dozierenden als fähig einschätzen, ein digitales Lehrangebot umzusetzen. Die selbsteingeschätzte Kompetenz zur Umsetzung der Online-Lehre wurde über insgesamt acht Items (angelehnt an Gosselin 2009, Beispiel: «Ich bin zuversichtlich, dass ich fähig bin, digitale Tools zu verwenden, um die Qualität meiner Lehre zu sichern») erfasst, die mit einer LikertSkala von 1 = «stimmt gar nicht» bis 4 = «stimmt völlig» beantwortet wurden. In den Analysen zeigte sich eine zufriedenstellende interne Konsistenz (Cronbach's $\alpha=0.83$ ).

Die erhobenen Daten wurden auf Ebene der einzelnen Items deskriptiv ausgewertet. Mithilfe non-parametrischer Verfahren wurden Unterschiede im Nutzungsverhalten digitaler Tools (asymptotischer Wilcoxon-Test) sowie Zusammenhänge des Nutzungsverhaltens mit dem Geschlecht der Dozierenden und deren selbsteingeschätzter Fähigkeit zur Umsetzung der Online-Lehre (Chi-Quadrat-Test und Rangkorrelationsanalyse) geprüft. Anhand von Mittelwertvergleichen (t-test und Varianzanalyse) wurden zudem Unterschiede zwischen weiblichen und männlichen Dozierenden sowie zwischen unterschiedlichen Altersgruppen hinsichtlich ihrer Fähigkeitsselbsteinschätzung und ihrem Nutzungsverhalten geprüft. Bei allen Analysen wurde das Signifikanzniveau auf $5 \%$ fixiert.

\section{Ergebnisse}

\subsection{Erfahrungen und Einschätzungen zur Umstellung auf Online-Lehre}

Die Ergebnisse der Befragung von Dozierenden aus Deutschland verdeutlichen ambivalente Erfahrungen und Einschätzungen in Bezug auf die Umstellung der Präsenzlehre auf Online-Lehre in Zeiten des Coronavirus-bedingten ersten Lockdowns der Universitäten. Gefragt nach den ersten Gedanken zur Umstellung stimmt knapp die Hälfte der Befragten (47.9 \%) der Aussage zu, dass sie online lehren können, dieses Lehrformat jedoch weniger zufriedenstellend sei. Dennoch sehen die meisten Befragten die Umstellung als eine Gelegenheit, den Umgang mit digitalen Tools zu erlernen (46.2 \%) und pädagogische Innovationen in ihrer Lehre umzusetzen (31.5\%) - wenngleich einige Dozierende (24.7\%) befürchten, dass die Studierenden auf die Herausforderung der Online-Lehre nicht vorbereitet seien. 
Insgesamt wird die Umstellungserfahrung von den meisten Dozierenden positiv wahrgenommen: So erfährt die Aussage, dass die Umstellung der Lehre eine überwiegend positive und ermutigende Erfahrung war, den grössten Zuspruch unter den Befragten (49.7\%). Zugleich wird die Umstellung von einigen Dozierenden (33.9\%) jedoch auch als eine komplexe Erfahrung beschrieben, die ein erhöhtes Mass an Investitionen zur Bewältigung erforderte. Nur vereinzelt wird die Umstellungserfahrung als frustrierend (13.4\%) oder überfordernd eingeschätzt (10.6\%).

Die Dozierenden scheinen insgesamt zuversichtlich, auch unter den aktuellen Bedingungen erfolgreich zu lehren. Für die selbsteingeschätzte Fähigkeit zur Umsetzung der Online-Lehre zeigt sich ein durchschnittlicher Skalenwert von $M=3.06$ (SD = 0.48). Auch der Vorbereitungsprozess und die ersten realisierten digitalen Lehrveranstaltungen werden von den Dozierenden überwiegend positiv eingeschätzt (vgl. Tab. 2). Nur vereinzelt werden die Vorbereitung und Umsetzung der ersten Lehreinheiten als schwierig bzw. nicht erfolgreich wahrgenommen; knapp ein Fünftel der Befragten räumt sich Verbesserungspotenzial bei der Durchführung der Online-Lehre ein. Anhand der offenen Antworten der Dozierenden wird deutlich, dass insbesondere der Zeitaufwand für die Vorbereitung und Durchführung der Online-Lehre als hoch und die eingeschränkte Interaktion mit den Studierenden als Schwierigkeit empfunden werden (z. B. fehlendes Feedback, kaum Redebeiträge).

\begin{tabular}{|l|c|c|}
\hline \multicolumn{2}{|c|}{$n$} & n \\
\hline Vorbereitungsprozess & 46 & 15.8 \\
\hline sehr einfach & 75 & 25.7 \\
\hline einfach & 106 & 36.3 \\
\hline adäquat & 32 & 11.0 \\
\hline schwierig & 3 & 1.0 \\
\hline sehr schwierig & 282 & 96.6 \\
\hline Total & & 1.4 \\
\hline Umsetzung erster Lehreinheiten & 4 & 19.9 \\
\hline nicht erfolgreich & 58 & 28.4 \\
\hline könnte besser sein & 83 & 31.5 \\
\hline angemessen & 92 & 5.1 \\
\hline erfolgreich & 15 & 90.8 \\
\hline sehr erfolgreich & 265 & \\
\hline Total & & \\
\hline
\end{tabular}

Tab. 2.: Vorbereitungsprozess und Umsetzung der Online-Lehre (Angabe Prozentwerte). 


\subsection{Nutzung digitaler Tools vor und während des Coronavirus-bedingten ersten Lockdowns}

Die vorliegenden Umfrageergebnisse verdeutlichen weiterhin, dass vor Ausbruch der Corona-Pandemie der Einsatz digitaler Tools in der Hochschullehre kaum verbreitet war bzw. sich auf gängige Formate, z. B. Präsentationstools, fokussierte. Mehr als die Hälfte der Befragten gibt an, vor dem ersten Lockdown der Universitäten digitale Tools gar nicht (11.3\%) oder nur in geringem Umfang (43.5\%) in den eigenen Lehrveranstaltungen eingesetzt zu haben. Die restlichen Befragten integrierten digitale Tools in mässigem (38.0 \%) bzw. grossem Umfang (6.5\%). Gründe für den Verzicht auf digitale Tools sind aus Sicht der Dozierenden der hohe Zeitaufwand zur Vorbereitung digitaler Lehrformate sowie das Erfordernis zusätzlicher personaler Ressourcen zur Unterstützung der Vorbereitung. Weiterhin geben die Dozierenden an, vor Ausbruch der Pandemie vor allem Learning Management System (LMS)-Plattformen wie beispielsweise Moodle in mässigem (25.0 \%) bzw. grossem Umfang (63.7 \%) eingesetzt zu haben, um Materialien und Literatur bereitzustellen. Weiterhin wurden vorrangig Präsentationen (z. B. unter Rückgriff auf PowerPoint) oder fremdproduzierte Videos (z. B. von YouTube) in die Lehre integriert, wohingegen selbstproduzierte Video von der Mehrheit der Befragten vor Ausbruch der Pandemie nicht genutzt wurden (77.1\%). In ähnlicher Weise stimmen 246 Dozierende (84.2 \%) der Aussage zu, Webkonferenzsysteme (z. B. Zoom) bislang nicht in ihrer Lehre eingesetzt zu haben.

Mithilfe non-parametrischer Verfahren wurde geprüft, inwieweit sich die Nutzung digitaler Tools vor und während des ersten Lockdowns unterscheidet. Mit Ausnahme von fremdproduzierten Videos werden für die Tools bedeutsame Zuwächse bzgl. des Einsatzes in mässigem und grossem Umfang deutlich (vgl. Tab. 3). Zwar überwiegen noch immer die Einbindung von LMS-Plattformen für Unterlagen und Bibliografie und die Bereitstellung von Präsentationen, allerdings erfahren während des ersten Lockdowns insbesondere der Einsatz von Webkonferenzsystemen zur Realisierung synchroner Online-Lehre sowie die Nutzung von LMS-Plattformen zur Ermöglichung von Diskussionsforen und Gruppenarbeiten starke Zuwächse. Auch selbstproduzierte Videos werden in Zeiten des ersten Lockdowns verstärkt in die Lehre integriert. 


\begin{tabular}{|c|c|c|c|}
\hline & $\begin{array}{l}\text { Vor erstem Lockdown } \\
\quad(n=277-289)\end{array}$ & $\begin{array}{l}\text { Während erstem Lock- } \\
\text { down }(n=274-287)\end{array}$ & Teststatistiken \\
\hline $\begin{array}{l}\text { LMS-Plattform für } \\
\text { Unterlagen und Bib- } \\
\text { liografie }\end{array}$ & $88.7 \%$ & $93.8 \%$ & $\begin{array}{c}z=-7.06 \\
p<.001 \\
n=284\end{array}$ \\
\hline $\begin{array}{l}\text { LMS-Plattform für } \\
\text { Diskussionsforen und } \\
\text { Gruppenarbeiten }\end{array}$ & $20.9 \%$ & $60.3 \%$ & $\begin{array}{c}z=-10.89 \\
p<.001 \\
n=265\end{array}$ \\
\hline $\begin{array}{l}\text { Präsentationen (z. B. } \\
\text { PowerPoint) }\end{array}$ & $81.8 \%$ & $85.6 \%$ & $\begin{array}{c}z=-2.62 \\
p<.01 \\
n=277\end{array}$ \\
\hline $\begin{array}{l}\text { Ausgewählte Videos } \\
\text { von YouTube oder } \\
\text { anderen }\end{array}$ & $42.8 \%$ & $47.9 \%$ & $\begin{array}{c}z=-1.59 \\
p=.112 \\
n=273\end{array}$ \\
\hline $\begin{array}{l}\text { Selbst produzierte } \\
\text { Videos }\end{array}$ & $10.6 \%$ & $28.7 \%$ & $\begin{array}{l}z=-7.83 \\
p<.001 \\
n=265\end{array}$ \\
\hline $\begin{array}{l}\text { Synchrone Online- } \\
\text { Lehre über Zoom oder } \\
\text { andere Tools }\end{array}$ & $7.2 \%$ & $68.5 \%$ & $\begin{array}{c}z=-12.64 \\
p<.001 \\
n=276\end{array}$ \\
\hline
\end{tabular}

Tab. 3.: Nutzung digitaler Tools in mässigem und grossem Umfang (Angabe Prozentwerte und Teststatistiken).

\subsection{Zusammenhänge zwischen personenbezogenen Merkmalen, Kompetenzeinschätzung und Nutzungsverhalten in Bezug auf digitale Tools}

Die Befunde der korrelativen Analysen (vgl. Tab. 4) verdeutlichen zunächst einen signifikanten Zusammenhang zwischen dem Nutzungsverhalten hinsichtlich digitaler Tools ${ }^{2}$ vor und während des ersten Lockdowns der Universitäten. Für den Einsatz digitaler Tools vor Ausbruch der Pandemie zeigen sich schwache Korrelationen mit dem Geschlecht und der Kompetenzeinschätzung bzgl. der Umsetzung von OnlineLehre, wobei nur Letztere statistisch signifikant wird. Für den Einsatz digitaler Tools während des ersten Lockdowns werden ebenfalls schwache, jedoch jeweils signifikante Korrelationen mit den personenbezogenen Merkmalen deutlich (vgl. Tab. 4).

2 Für die korrelativen Analysen wurden die Skalen «Nutzungsverhalten vor Lockdown» und «Nutzungsverhalten während Lockdown» über Mittelwertbildung der jeweiligen Einzelitems entwickelt. 


\begin{tabular}{|c|c|c|c|c|}
\hline & $(1)$ & $(2)$ & (3) & (4) \\
\hline Geschlecht $^{1}(1)$ & 1 & & & \\
\hline $\begin{array}{l}\text { Selbsteingeschätzte Kompetenz zur Umsetzung } \\
\text { von Online-Lehre (2) }\end{array}$ & $0.186^{*}$ & 1 & & \\
\hline Nutzungsverhalten vor dem ersten Lockdown (3) & 0.084 & $0.287^{\star \star}$ & 1 & \\
\hline $\begin{array}{l}\text { Nutzungsverhalten während des ersten Lock- } \\
\text { downs (4) }\end{array}$ & $0.212^{\star \star}$ & $0.284^{\star \star}$ & $0.495^{\star \star}$ & 1 \\
\hline
\end{tabular}

Tab. 4.: Zusammenhänge zwischen Geschlecht, Kompetenzwahrnehmung und Nutzungsverhalten (Angabe von Rangkorrelationskoeffizienten nach Spearman ( $r s$ ) und Eta-Koeffizienten). Anmerkungen: ${ }^{1}$ Angabe von Eta-Koeffizienten, Codierung: $1=$ männlich; ${ }^{\star \star} p$ $<.01,{ }^{\star} \mathrm{p}<.05$.

Um die korrelativen Befunde weiter zu beleuchten, wurden Mittelwertvergleiche realisiert. Das Ausmass der Nutzung digitaler Tools während des ersten Lockdowns unterscheidet sich zwischen den Geschlechtern $(t(274)=3.593, p<0.001, d=0.512)$. Die weiblichen Dozierenden $(n=175)$ setzten demnach im Sommersemester 2020 in höherem Ausmass digitale Tools in ihrer Lehre ein $(M=2.89, S D=0.51)$ als die männlichen Befragten $(M=2.66, S D=0.51, n=102)$. Bereits vor dem ersten Lockdown der Universitäten war das generelle Nutzungsverhalten bzgl. digitaler Tools bei den weiblichen Dozierenden $(M=2.13, S D=0.48)$ etwas höher ausgeprägt als bei den Männlichen $(M=2.05, S D=0.43)$, jedoch ist dieser Unterschied nicht signifikant $(t(275)=1.392, p=0.165, d=0.465)$. Auch die selbsteingeschätzte Kompetenz, digital zu lehren, ist bei den weiblichen Dozierenden $(M=3.14, S D=0.41$,) geringfügig höher ausgeprägt ist als bei den männlichen Dozierenden $(M=2.96, S D=0.53)$. Der Gruppenunterschied erweist sich bei mittlerer Effektstärke als statistisch signifikant $(t(172.034)=2.938, p=0.004, d=0.460)$. Für Dozierende unterschiedlicher Altersgruppen werden im Rahmen der Varianzanalysen keine Unterschiede hinsichtlich ihrer selbsteingeschätzten Kompetenz, digital zu lehren $(F(3,283)=2.011, p=0.113)$, dem Ausmass der Nutzung digitaler Tools vor der Coronavirus-bedingten Lehrsituation $(F(3,283)=0.139, p=0.937)$ sowie in Hinblick auf das Nutzungsverhalten während des ersten Lockdowns der Universitäten $(F(3,282)=0.878, p=0.453)$, ersichtlich.

\section{Diskussion}

Während bisherige Befunde der CRTS-Studie die institutionsspezifischen Erfahrungen von Dozierenden in den Blick nehmen, ermöglichen die vorliegenden Ergebnisse anhand einer Stichprobe, die Dozierende von insgesamt 42 Hochschulen in Deutschland umfasst, eine Betrachtung der Umstellung auf Online-Lehre über verschiedene Hochschulstandorte hinweg. Im Einklang mit Befunden der CRTS-Studie für die Hochschulstandorte Duisburg-Essen, Basel (Göbel et al. 2021), Jerusalem (Dorfsman und Horenczyk 2021) und Buenos Aires (Lion et al. eingereicht) weisen 
auch die präsentierten Befunde auf eine überwiegend erfolgreiche Bewältigung der Herausforderungen, die sich durch den ersten Lockdown deutscher Hochschulen zu Beginn der Corona-Pandemie ergeben haben, hin: Sowohl die Vorbereitung als auch die Durchführung der ersten digitalen Lehreinheiten werden von den Befragten mehrheitlich als gelungen bewertet und die Lehrenden sind zuversichtlich, trotz der digitalen Lehrsituation ihre Lehrziele zu erreichen. Die positiven Einschätzungen der Dozierenden stimmen auch mit weiteren Befunden zur Wahrnehmung der Hochschullehre zu Beginn der Corona-Pandemie überein (z. B. Dittler und Kreidl 2021; Almazova et al. 2020; Kienle und Appel 2021; Watermeyer et al. 2020) und es wird deutlich, dass die befragten Hochschulangehörigen die Potenziale der Digitalisierung im Sinne der Einführung didaktischer Innovationen und der Entwicklung digitaler Kompetenzen durchaus erkennen (Getto, Hintze, und Kerres 2018).

Analog zu bisherigen Erkenntnissen (z. B. Schmid et al. 2017) wurden vor Ausbruch der Pandemie von den befragten Dozierenden vorranging Präsentationstools oder LMS-Plattformen zur Bereitstellung von z. B. Literatur in die eigene Lehre integriert, während synchrone Formate wie Webinare oder Videokonferenzen nur selten eingesetzt wurden (Schaarschmidt, Albrecht, und Börner 2016). Die vorgelegten Befunde zeigen während des ersten Lockdowns der Universitäten einen Anstieg im Nutzungsverhalten digitaler Tools, vor allem im Zusammenhang mit Webkonferenzsystemen und LMS-Plattformen zur Ermöglichung kooperativer Arbeitsweisen. Auch aus Studierendensicht wird ein vermehrter Einsatz derartiger Formate im Sommersemester 2020 bekundet (Karapanos et al. 2021). Wenngleich die verstärkte Nutzung digitaler Tools, und speziell von synchronen Werkzeugen wie Videokonferenzen (Kienle und Appel 2021; Lowenthal et al. 2020), aufgrund der Schliessung der Universitäten erwartbar war, verdeutlichen die Befunde, dass die Hochschulen eine angemessene technologische Infrastruktur zur Verfügung stellen konnten, und es den Dozierenden vermeintlich gelungen scheint, eine zeit- und ortsunabhängige Auseinandersetzung mit den Lerninhalten anzubieten (Kümmel et al. 2020).

In Ergänzung zu den bisherigen Erkenntnissen der CRTS-Studie weisen die vorliegenden Befunde der Korrelationsanalysen auf die Relevanz von Vorerfahrungen (bzw. bisheriger Nutzung digitaler Tools), Kompetenzeinschätzung in Bezug auf Online-Lehre und Geschlecht für das Ausmass des Einsatzes digitaler Tools in der Lehre hin. So liegen in der vorliegenden Stichprobe schwache positive Zusammenhänge zwischen der Kompetenzselbsteinschätzung der Dozierenden und dem Ausmass der Nutzung digitaler Tools sowohl vor als auch während des ersten Lockdowns der Universitäten vor. Diese unterstützen bisherige Befunde, nach denen für den Einsatz digitaler Tools in der Hochschule neben technologischer Infrastruktur mitunter auch digitale Kompetenzen der Lehrenden erforderlich sind (Buchanan 2013; Jokiaho und May 2017). Dass auch Vorerfahrungen im Umgang mit digitalen Medien für das eigene 
Nutzungsverhalten von Bedeutung sein können, verdeutlicht der vorliegende Zusammenhang zwischen dem Ausmass der Nutzung digitaler Tools vor und während des ersten Lockdowns. Weiterhin finden sich Unterschiede zwischen den Geschlechtern, welche vor dem Hintergrund bisheriger Befunde zu Digitalisierungsprozessen in Schulen (Drossel et al. 2019) und Hochschulen (Riedel und Börner 2016; Van der Spoel et al. 2020) nicht erwartet wurden. Während in bisherigen Studien Männern Vorteile im Umgang mit digitalen Medien und Kompetenzen zugesprochen wurden, weisen in den vorgelegten Analysen die weiblichen Dozierenden höhere Kompetenzeinschätzungen hinsichtlich der Umsetzung der Online-Lehre und ein höheres Nutzungsverhalten bzgl. digitaler Tools während des ersten Lockdowns auf als die Männer. Für den schulischen Kontext konnten bisherige Untersuchungen Unterschiede zwischen Lehrkräften unterschiedlichen Alters hinsichtlich ihrer Einstellung gegenüber Mediennutzung aufzeigen (Drossel et al. 2019), welche sich in unseren Daten nicht widerspiegeln.

Wenngleich die Zusammenschau eine erfolgreiche Bewältigung der Umstellung auf digitale Lehr-Lernformate im Frühjahr 2020 signalisiert, werden analog zu aktuellen Befunden zur Lehrsituation der Hochschulen (Almazova et al. 2020; Göbel et al. 2021; Kienle und Appel 2021) auch in der vorliegenden Stichprobe ambivalente Einschätzungen zur Online-Lehre deutlich. Zwar wird der Erfolg der ersten Lehreinheiten während des ersten Lockdowns der Universitäten überwiegend positiv bewertet, zugleich schätzen viele Dozierende digitale Lehrformate im Vergleich zur Präsenzlehre jedoch als weniger zufriedenstellend ein und beschreiben die Umstellungserfahrung als komplexe Anforderung mit einem erhöhten Mass an Investitionen. Dabei werden von den Befragten insbesondere der mit der Vorbereitung und Durchführung der digitalen Lehre verbundene zeitliche Aufwand als Belastungsfaktor genannt (Schmid et al. 2017; Voß und Raichle 2016) und der fehlende Austausch mit Studierenden als einschränkend erlebt. Auch die Befunde bisheriger Studierendenbefragungen verdeutlichen, dass der erhöhte Workload und fehlende Kontakt zu Dozierenden und Peers bei den Studierenden Gefühle der Verzweiflung und Überforderung auslösen können (Brunner 2021; Karapanos et al. 2021; Stammen und Ebert 2020; Zierer 2020). Konnte die Implementation digitaler Lehre im Zuge des ersten Lockdowns im Frühjahr 2020 bedingt durch die sofortige Umstellung als «Notfalllehre» (vgl. Hodges et al. 2020) gelten, ist es aufgrund der aktuellen Entwicklung der Pandemie und der mehrheitlich anhaltenden Distanzlehre nun an der Zeit, über Notfallpraktiken hinauszugehen und sorgfältig geplante Ansätze für digitale Lehr-Lernformate anzubieten. Die aktuelle Befundlage weist auf den Bedarf einer konzeptionellen Weiterentwicklung digitaler Lehrformate insbesondere in Bezug auf soziale und kollaborative Komponenten des Lernens hin. Mit Blick auf die Dozierenden sind die Hochschulen - auch vor dem Hintergrund der pandemie-bedingten Einschränkungen über den Beruf hinausgehend ( $z$. B. Fürsorge-Aufgaben oder psychische Beschwerden) - mehr denn 
je gefordert, noch breitere Unterstützungs- und Qualifizierungsangebote anzubieten und Arbeitsbedingungen zu schaffen, welche erfolgreiche Online-Lehre unterstützen und positive Einstellungen diesbezüglich befördern bzw. aufrechterhalten können.

Kritisch zu bemerken ist, dass aufgrund der Freiwilligkeit der Teilnahme an der CRTS-Studie Selbstselektionsprozesse zu einer Verzerrung der Stichprobe geführt haben könnten. Unter Umständen haben vorranging Dozierende teilgenommen, die eine generell positive Haltung gegenüber der Digitalisierung von Studium und Lehre aufweisen; hingegen wurden Dozierende, die zum Erhebungszeitpunkt aufgrund der veränderten beruflichen wie auch privaten Gegebenheiten ein erhöhtes Belastungserleben aufweisen, eventuell in geringerem Masse erreicht. Kritisch anzumerken ist zudem, dass es sich ausschliesslich um Selbstauskünfte der Hochschullehrenden handelt, die durch Prozesse der sozialen Erwünschtheit beeinflusst gewesen sein könnten. Insgesamt kann bei der vorliegenden Stichprobe nicht von einer Repräsentativität für die Dozierenden in Deutschland ausgegangen werden. Es bedarf entsprechend weiterer Untersuchungen, um die aufgezeigten Korrelationen zwischen personenbezogenen Merkmalen, Kompetenzeinschätzungen und Nutzung digitaler Tools in der Hochschullehre zu bestätigen. In diesem Zusammenhang sollten über die bereits eingesetzten Instrumente auch persönliche Nützlichkeitsempfindungen in den Blick genommen werden, da sich diese in der Technologie-Akzeptanz-Forschung als für das Nutzungsverhalten digitaler Medien relevant erwiesen haben (Davis 1993, Teo 2009). Um langfristige Wirkungen in den Blick zu nehmen und aufzuklären, inwieweit die aktuelle Umstellung auf Online-Lehre zu einer Weiterentwicklung der didaktischen Qualität der Lehre beitragen kann, sind darüber hinaus weiterführende experimentelle und längsschnittlich angelegte Studien wünschenswert.

Trotz der genannten Einschränkungen ermöglichen die präsentierten Befunde interessante Einblicke in die pandemie-bedingte Umstellung der Lehre über verschiedene Hochschulstandorte hinweg und können dadurch wertvolle Hinweise für die weiterführende Digitalisierung von Studium und Lehre liefern, welche auch nach der Corona-Pandemie Bestand haben kann. Im weiteren Verlauf des CRTS-Projekts ist die Zusammenführung der in den beteiligten Ländern generierten Fragebogendaten angestrebt, um ländervergleichende Perspektiven einzunehmen. Dabei sollen die Relevanz personenbezogener Aspekte (z. B. Geschlecht und Alter) für den Einsatz digitaler Technologien in der eigenen Lehre im Zuge des ersten Lockdowns der Universitäten ländervergleichend untersucht, sowie mögliche Unterschiede zwischen den Dozierenden unterschiedlicher Fachbereiche und Lehrdeputate geprüft werden. 


\section{Literatur}

Ackeren, Isabell van, Michael Kerres, und Sandrina Heinrich. 2017. Flexibles Lernen mit digitalen Medien. Strategische Verankerung und Handlungsfelder an der Universität DuisburgEssen. Münster: Waxmann. https://doi.org/10.25656/01:15385.

Almazova, Nadezhda, Elena Krylova, Anna Rubtsova, und Maria Odinokaya. 2020. «Challenges and Opportunities for Russian Higher Education amid COVID-19: Teachers' Perspective». Educ. Sci. 10 (12): 368. https://doi.org/10.3390/educsci10120368.

Bandura, Albert. 1977. «Self-efficacy: Toward a unifying theory of behavioral change». Psychological Review 84: 191-215. https://doi.org/10.1037/0033-295X.84.2.191.

Bao, Wei. 2020. «COVID-19 and online teaching in higher education: A case study of Peking University». Human Behavior and Emerging Technologies 2 (2): 113-115. https://onlinelibrary. wiley.com/doi/epdf/10.1002/hbe2.191.

Bozkurt, Aras, und Ramesh C. Sharma. 2020. «Emergency remote teaching in a time of global crisis due to CoronaVirus pandemic». Asian Journal of Distance Education 15 (1): 1-5. http:// www.asianjde.com/ojs/index.php/AsianJDE/article/view/447.

Brunner, Georg. 2021. «Das Corona-Semester - die Zwangsumstellung auf Fernlehre aus Sicht der Hochschulleitung am Beispiel der Pädagogischen Hochschule Freiburg». In Wie Corona die Hochschullehre verändert. Erfahrungen und Gedanken aus der Krise, herausgegeben von Ulrich Dittler, und Christian Kreidl, 71-88. Wiesbaden: Springer Gabler. https://link.springer.com/book/10.1007/978-3-658-32609-8.

Bundesregierung. 2018. Ein neuer Aufbruch für Europa. Eine neue Dynamik für Deutschland. Ein neuer Zusammenhalt für unser Land. Koalitionsvertrag zwischen CDU, CSU und SPD (19. Legislaturperiode). https://www.bundestag.de/resource/blob/543200/9f9f21a92a618c77aa3 30f00ed21e308/kw49_koalition_koalitionsvertrag-data.pdf.

Buchanan, Tom, Phillip Sainter, und Gunter Saunders. 2013. «Factors affecting faculty use of learning technologies: implications for models of technology adoption». Journal of Computing in Higher Education 25: 1-11. https://doi.org/10.1007/s12528-013-9066-6.

Carrillo, Carmen, und Maria Assunção Flores. 2020. «COVID-19 and teacher education: a literature review of online teaching and learning practices». European Journal of Teacher Education 43 (4): 466-487. https://doi.org/10.1080/02619768.2020.1821184.

Czerniewicz, Laura, et al. 2020. «A Wake-Up Call: Equity, Inequality and Covid-19 Emergency Remote Teaching and Learning». Postdigital Science and Education 2: 946-967. https://doi. org/10.1007/s42438-020-00187-4.

Davis, Fred D. 1989. «Perceived Usefulness, Perceived Ease of Use, and User Acceptance of Information Technology». MIS Quarterly 13 (3): 319-340. https://www.jstor.org/stable/249008.

Davis, Fred D. 1993. «User acceptance of information technology: system characteristics, user perceptions and behavioral impacts». International Journal of Man-Machine Studies 38 (3): 475-487. https://doi.org/10.1006/imms.1993.1022. 
Dittler, Ulrich, und Christian Kreidl. 2021. Wie Corona die Hochschullehre verändert. Erfahrungen und Gedanken aus der Krise zum künftigen Einsatz von eLearning. Wiesbaden: Springer Gabler. https://link.springer.com/book/10.1007/978-3-658-32609-8.

Dorfsman, Marcelo, und Gabriel Horenczyk. 2021. «The Coping of Academic Staff with an Extreme Situation: The Transition from Conventional Teaching to Online Teaching». Education and Information Technologies, Juli. https://doi.org/10.1007/s10639-021-10675-0.

Dräger, Jörg, und Frank Ziegele. 2014. Hochschulbildung wird zum Normalfall. Ein gesellschaftlicher Wandel und seine Folgen. CHE - Centrum für Hochschulentwicklung. https://www. che.de/download/hochschulbildung_wird_zum_normalfall_2014-pdf.

Drossel, Kerstin, Birgit Eickelmann, Heike Schaumburg, und Amelie Labusch. 2019. "Nutzung digitaler Medien und Prädiktoren aus der Perspektive der Lehrerinnen und Lehrer im internationalen Vergleich». In ICILS 2018 \#Deutschland. Computer- und informationsbezogene Kompetenzen von Schülerinnen und Schülern im zweiten internationalen Vergleich und Kompetenzen im Bereich Computational Thinking, herausgegeben von Birgit Eickelmann, Wilfried Bos, Julia Gerick, Frank Goldhammer, Heike Schaumburg, Knut Schwippert, Knut, Martin Senkbeil, und Jan Vahrenhold, 205-240. Münster: Waxmann. https://doi. org/10.25656/01:18325.

Education International. 2020. Guiding Principles on the COVID-19 Pandemic. https://www.ei-ie. org/en/detail/16701/guiding-principles-on-the-covid-19-pandemic.

Ferdig, Richard E., Emily Baumgartner, Ricard Hartshorne, Regina Kaplan-Rakowski, und Chrystalla Mouza. 2020. Teaching, Technology, and Teacher Education During the COVID-19 Pandemic: Stories from the Field. Association for the Advancement of Computing in Education (AACE). https://www.learntechlib.org/p/216903/.

Flores, Maria Assunção, und Marília Gago. 2020. «Teacher education in times of COVID-19 pandemic in Portugal: national, institutional and pedagogical responses». Journal of Education for Teaching 46 (4): 507-516. https://doi.org/10.1080/02607476.2020.1799709

Getto, Barbara, Patrick Hintze, und Michael Kerres. 2018. «(Wie) Kann Digitalisierung zur Hochschulentwicklung beitragen?»In Digitalisierung und Hochschulentwicklung. Proceedings zur 26. Tagung der Gesellschaft für Medien in der Wissenschaft e.V., herausgegeben von Barbara Getto, Patrick Hintze, und Michael Kerres, 13-25. Münster: Waxmann. https://www.pedocs.de/volltexte/2019/16983/pdf/MidW_74_Getto_et_al_Wie_kann_Digitalisierung.pdf

Getto, Barbara, und Katrin Schulenburg. 2018. «Digitalisierung im Kontext strategischer Hochschulentwicklung an den Hochschulen in Nordrhein-Westfalen». In Digitalisierung und Hochschulentwicklung. Proceedings zur 26. Tagung der Gesellschaft für Medien in der Wissenschaft e.V., herausgegeben von Barbara Getto, Patrick Hintze, und Michael Kerres, 36-96. Münster: Waxmann. https://doi.org/10.25656/01:16998.

Gilch, Harald, Anna Sophie Beise, René Krempkow, Marko Müller, Friedrich Stratmann, und Klaus Wannemacher. 2019. Digitalisierung der Hochschulen. Ergebnisse einer Schwerpunktstudie für die Expertenkommission Forschung und Innovation. Expertenkommission Forschung und Innovation (EFI). Berlin. https://his-he.de/publikationen/detail/digitalisierung-der-hochschulen. 
Göbel, Kerstin, Elena Makarova, Katharina Neuber, und Tomas Kaqinari. 2021. «Der Übergang zur digitalen Lehre an den Universitäten Duisburg-Essen und Basel in Zeiten der CoronaPandemie». In Wie Corona die Hochschullehre verändert. Erfahrungen und Gedanken aus der Krise, herausgegeben von Ulrich Dittler, und Christian Kreidl, 351-374. Wiesbaden: Springer Gabler. https://doi.org/10.1007/978-3-658-32609-8.

Gosselin, Kevin P. 2009. Development and psychometric exploration of the online teaching selfefficacy inventory. Texas Tech University, Lubbock. https://ttu-ir.tdl.org/bitstream/handle/2346/8971/Gosselin_Kevin_Diss.pdf.

Greimel-Fuhrmann, Bettina, Julia Riess, Tim Loibl, und Susanne Schuster. 2021. «Lehren aus der Distanzlehre ziehen - eine Interviewstudie zur Distanzlehre an der Wirtschaftsuniversität Wien». In Wie Corona die Hochschullehre verändert. Erfahrungen und Gedanken aus der Krise, herausgegeben von Ulrich Dittler, und Christian Kreidl, 89-104. Wiesbaden: Springer Gabler. https://doi.org/10.1007/978-3-658-32609-8.

Handke, Jürgen. 2020. Handbuch Hochschullehre Digital. Leitfaden für eine moderne und mediengerechte Lehre. 3., aktualisierte und erweiterte Auflage. Baden-Baden: Tectum Verlag. https://doi.org/10.5771/9783828875302.

Hodges, Charles, Stephanie Moore, Barb Lockee, Torrey Trust, und Aaron Bond. 2020. "The difference between emergency remote teaching and online learning». EDUCAUSE Review. https://er.educause.edu/articles/2020/3/the-difference-between-emergency-remoteteaching-and-online-learning.

Jokiaho, Annika, und Birgit May. 2017. «Hindernisse für die Nutzung von E-Learning an Hochschulen. Aktueller Forschungsstand». In Bildungsräume. Proceedings der 25. Jahrestagung der Gesellschaft für Medien in der Wissenschaft, 5. bis 8. September 2017 in Chemnitz, herausgegeben von Christoph Igel, 20-31. Münster: Waxmann. https://doi.org/10.25656/01:16107.

Karapanos, Marios, Robert Pelz, Patrick Hawlitschek, und Heinz-Werner Wollersheim. 2021. «Hochschullehre im Pandemiebetrieb. Wie Studierende in Sachsen das digitale Sommersemester erlebten». MedienPädagogik 40 (CoViD-19): 1-24. https://doi.org/10.21240/ mpaed/40/2021.01.28.X.

Kehrer, Mareike, und Anne Thillosen. 2021. «Hochschulbildung nach Corona - ein Plädoyer für Vernetzung, Zusammenarbeit und Diskurs». In Wie Corona die Hochschullehre verändert. Erfahrungen und Gedanken aus der Krise, herausgegeben von Ulrich Dittler, und Christian Kreidl, 51-70. Wiesbaden: Springer Gabler. https://doi.org/10.1007/978-3-658-32609-8.

Kerres, Michael. 2016. «E-Learning oder Digitalisierung in der Bildung: Neues Label oder neues Paradigma?» Grundlagen der Weiterbildung - Praxishilfen 7: 159-171. https://learninglab. uni-due.de/sites/default/files/GdWB\%20elearning-vs-digitalisierung.pdf.

Kienle, Andrea, und Tamara Appel. 2021. «In 25 Tagen in die digitale Welt: Das Online-Semester an der Fachhochschule Dortmund». In Wie Corona die Hochschullehre verändert. Erfahrungen und Gedanken aus der Krise, herausgegeben von Ulrich Dittler, und Christian Kreidl, 105-118. Wiesbaden: Springer Gabler. https://doi.org/10.1007/978-3-658-32609-8. 
Kümmel, Elke, Johannes Moskaliuk, Ulrike Cress, und Joachim Kimmerle. 2020. «Digital Learning Environments in Higher Education: A Literature Review of the Role of Individual vs. Social Settings for Measuring Learning Outcomes». Educ. Sci. 10 (3): 78. https://doi. org/10.3390/educsci10030078.

Lion, Carina, Uriel Cukierman, und Mónica Scardigli, Mónica. Submitted. «The emergence of the emergency in Higher Education in Argentina». In The changing character of universities: the impact of the COVID19 pandemic, herausgegeben von Martha Burkle and Alan Tait.

Lowenthal, Patrick R., Jered Borup, Richard E. West, und Leanna Archambault. 2020. «Thinking Beyond Zoom: Using Asynchronous Video to Maintain Connection and Engagement During the COVID-19 Pandemic». Journal of Technology and Teacher Education 28 (2): 383-391. https://www.learntechlib.org/primary/p/216192/.

Marzilli, Colleen, Julie Delello, Shelly Marmion, Rochell McWhorter, Paul Roberts, und T. Scott Marzilli. 2014. "Faculty Attitudes towards integrating Technology and Innovation». International Journal on Integrating Technology in Education 3 (1). https://arxiv.org/ftp/arxiv/ papers/1404/1404.4334.pdf.

Moorhouse, Benjamin Luke. 2020. "Adaptations to a face-to-face initial teacher education course 'forced' online due to the COVID-19 pandemic». Journal of Education for Teaching 46 (4). https://doi.org/10.1080/02607476.2020.1755205.

Müller, Ralph, Michael Eichhorn, und Alexander Tillmann. 2019. «Wie verändern sich E-Learning-Konzepte durch mediendidaktische Fortbildungen? Eine Längsschnittuntersuchung». In Teilhabe in der digitalen Bildungswelt, herausgegeben von Jörg Hafer, Martina Mauch, und Marle Schumann, 176-186. Waxmann: Münster. https://doi.org/10.25656/01:18022.

Mulders, Miriam, und Sophia Krah. 2021. «Digitales Lernen während der Covid-19-Pandemie aus Sicht von Studierenden der Erziehungswissenschaften. Handlungsempfehlungen für die Digitalisierung von Hochschullehre». MedienPädagogik 40 (CoViD-19): 25-44. https:// doi. org/10.21240/mpaed/40/2021.01.29.X.

Riedel, Jana, und Claudia Börner. 2016. «Wir tun es, weil es gut ist! Wie Lehrende die Erfolgsfaktoren für den Einsatz digitaler Medien in der Hochschullehre einschätzen». In Teaching Trends 2016. Digitalisierung in der Hochschule: Mehr Vielfalt in der Lehre, herausgegeben von Wolfgang Pfau, Caroline Baetge, Svenja Mareike Bedenlier, Carina Kramer, und Joachim Stöter, 209-220. Münster: Waxmann. https://doi.org/10.25656/01:18900.

Schaarschmidt, Nadine, Claudia Albrecht, und Claudia Börner. 2016. «Videoeinsatz in der Lehre. Nutzung und Verbreitung in der Hochschule». In Teaching Trends 2016. Digitalisierung in der Hochschule: Mehr Vielfalt in der Lehre, herausgegeben von Wolfgang Pfau, Caroline Baetge, Svenja Mareike Bedenlier, Carina Kramer, und Joachim Stöter, 39-48. Münster: Waxmann. https://doi.org/10.25656/01:18884. 
Schulze-Vorberg, Lukas, S. Franziska C. Wenzel, Claudia Bremer, und Holger Horz. 2018. «Die Öffnung von (Lern-)Räumen in Schule und Unterricht durch den Einsatz digitaler Medien. Der Einfluss von Computereinstellung, -ängstlichkeit und Lehrhaltung auf die digitale Mediennutzung von Lehrkräften». In Jahrbuch Medienpädagogik 14. Der digitale Raum - Medienpädagogische Untersuchungen und Perspektiven, herausgegeben von Manula Pietraß, Johannes Fromme, Petra Grell, und Theo Hug, 215-236. Wiesbaden: Springer. https://doi. org/10.1007/978-3-658-19839-8_12.

Stammen, Karl-Heinz, und Anna Ebert. 2020. Noch online? Studierendenbefragung zur medientechnischen Ausstattung im Sommersemester 2020. https://panel.uni-due.de/assets_websites/18/StammenEbert_2020_NochOnline_Gesamtbericht.pdf.

Teo, Timothy. 2009. «Modelling technology acceptance in education: A study of pre-service teachers». Computers \& Education 52: 302-312. https://doi.org/10.1016/j.compedu.2008.08.006.

Toquero, Cathy Mae. 2020. «Challenges and Opportunities for Higher Education amid the COVID-19 Pandemic: The Philippine Context». Pedagogical Research 5 (4). https://doi. org/10.29333/pr/7947.

UNESCO. 2020. COVID-19 Educational Disruption and Response. https://en.unesco.org/ node/320920

Van der Spoel, Irene, Omid Noroozi, Ellen Schuurink, und Stan van Ginkel. 2020. «Teachers' online teaching expectations and experiences during the Covid19-pandemic in the Netherlands». European Journal of Teacher Education 43 (4): 623-638. https://doi.org/10.1080/02 619768.2020.1821185.

Voß, Kerstin, und Nico Raichle. 2016. «Anreize, Motivation und Support für Lehrende zum Einsatz digitaler Medien in der Hochschullehre Ergebnisse einer qualitativen Expertenbefragung aus dem Verbundprojekt „work\&study“ - offene Hochschulen Rhein-Saar». In Teaching Trends 2016. Digitalisierung in der Hochschule: Mehr Vielfalt in der Lehre, herausgegeben von Wolfgang Pfau, Caroline Baetge, Svenja Mareike Bedenlier, Carina Kramer, und Joachim Stöter, 191-198. Münster: Waxmann. https://doi.org/10.25656/01:15136.

Wannemacher, Klaus, Imke Jungermann, Julia Scholz, Hacer Tercanli, und Anna von Villiez. 2016. Digitale Lernszenarien im Hochschulbereich. Im Auftrag der Themengruppe „Innovation in Lern- und Prüfungsszenarien "koordiniert vom CHE im Hochschulforum Digitalisierung. HIS-Institut für Hochschulentwicklung. https://www.che.de/wp-content/uploads/upload/ HFD_AP_Nr_15_Digitale_Lernszenarien.pdf.

Watermeyer, Richard, Tom Crick, Cathryn Knight, und Janet Goodall. 2020. «COVID-19 and digital disruption in UK universities: afflictions and affordances of emergency online migration». Higher Education 81: 623-641. https://doi.org/10.1007/s10734-020-00561-y.

Zhu, Xudong, und Jing Liu. 2020. «Education in and After Covid-19: Immediate Responses and Long-Term Visions». Postdigital Science and Education 2: 695-699. https://doi.org/10.1007/ s42438-020-00126-3.

Zierer, Klaus. 2020. «So viel Präsenz wie möglich. Einschätzungen von Studierenden zum Corona-Semester». Forschung \& Lehre 9: 755. https://wh2xhc1p6.homepage.t-online.de/. cm4all/uproc.php/0/forschung-und-lehre_9-2020.pdf. 